\title{
Improved Stability Criteria for Neural Networks with Additive Time-varying Delay Components
}

\author{
Jianmin Jiao \\ College of Mathematics and Information Science, Baoji University of \\ Arts and Sciences, Baoji, 721013, China \\ jmjiao@126.com
}

\begin{abstract}
This paper investigated the problem of improved delay-dependent stability criteria for neural networks with two additive time-varying delay components. By constructing a more general type of Lyapunov functional and using effective mathematical technique to estimate the upper bound on the time derivative of the Lyapunov functional, some new delay-dependent stability criteria are derived in terms of linear matrix inequalities. The resulting stability criteria are of fewer matrix variables and less conservative than some existing ones. Numerical examples are given to illustrate the effectiveness and less conservativeness of the proposed method.
\end{abstract}

Keywords: Neural networks, stability, additive time-varying delays, linear matrix inequality (LMI)

\section{Introduction}

In the past few decades, neural networks have been investigated extensively because of their successful applications in various scientific areas, such as pattern recognition, signal processing, image processing, associative memories, and so on [1,2]. It is well known that time delay is unavoidably existent in various real-world systems, and it is often a major cause of oscillation and instability [3-5]. Thus, the stability analysis for time delay neural networks has received considerable attention in recent years. The stability criteria for time delay neural networks can be classified into two types: delay-independent stability criteria [6-8] and delay-dependent stability criteria [9-25]. Generally speaking, the delaydependent stability criteria are less conservative than delay-independent ones, especially when the size of time-delays is small. Many efforts have been made to the research of delay-dependent stability problems for neural networks, and various methods are utilized for getting less conservative results, such as free weighting matrix method [9-13], augmented Lyapunov functional method [14-16], discretized Lyapunov functional method [17], delay-partitioning method [18-22], delay-slope-dependent method [23], and reciprocally convex method $[24,25]$. Note that the delay-dependent stability results in [625] can only provide stability conditions for neural networks with a single delay in the state. Recently, a new model for linear systems with two additive time-varying delays was proposed in [26]. The new model has a strong application background in networked control systems $[27,28]$. Some delay-dependent stability criteria for linear systems with two additive time-varying delay components are derived in [26-30]. In [31], Zhao et al. have initially investigated the globally asymptotic stability analysis of neural networks with two additive time-varying delay components by using the free-weighting matrix method. In [32,33], by constructing modified Lyapunov functionals, some improved delay-dependent stability criteria were obtained by using the convex polyhedron method. [34] and [35] have also considered the stability problem for neural networks with two additive time-varying delay components by constructing augmented Lyapunov functionals which contain triple integral terms, and some delay-dependent stability criteria 
were obtained by employing the reciprocally convex method and the delay-partitioning method, respectively. In [36], by defining a more general Lyapunov functional, a simplified delay-dependent stability criterion is established. Yet it is worth pointing out that, though the works above were elegant, there still exist some points waiting for the improvement. Firstly, the information of neuron activation function is not adequately considered, which may lead to much conservatism. Secondly, many matrix variables are introduced in the obtained results, which brings a large computational burden.

Motivated by the preceding discussions, by introducing a more general type of Lyapunov functional in this paper, some improved delay-dependent stability criteria for neural networks with two additive time-varying delay components are derived. Since more information of the time-varying delays are used, our method can get less conservative results compared to some existing literature. Furthermore, since reciprocally convex method is employed, the computational complexity is also reduced. The achieved stability criteria are expressed by a set of LMIs, which can be easily checked by Matlab LMI toolbox. Two numerical examples are provided to show the effectiveness and less conservativeness of the proposed method.

Notations: Throughout this paper, $\square$ "denotes the $n$-dimensional Euclidean space, while $\square^{n \times m}$ refers to the set of all real matrices with $m$ rows and $n$ columns. $A^{\mathrm{T}}$ represents the transpose of the matrix $A$. For real symmetric matrices $X$ and $Y$, the notation $X \geqslant Y$ (respectively, $X>Y$ ) means matrix $X-Y$ is positive-semidefinite (respectively, positive-definite). $I_{n}$ is the $n$-dimensional identity matrix.

\section{Problem Description and Preliminaries}

Consider the following delayed neural network with two additive time-varying delay components

$$
\dot{x}(t)=-C x(t)+A g(x(t))+B g\left(x\left(t-d_{1}(t)-d_{2}(t)\right)\right)+J,
$$

where $x(\cdot)=\left[x_{1}(\cdot), x_{2}(\cdot), \cdots, x_{n}(\cdot)\right]^{\mathrm{T}} \in \square^{n}$ is the state vector associated with the $n$ neurons, $g(x(\cdot))=\left[g_{1}\left(x_{1}(\cdot)\right), g_{2}\left(x_{2}(\cdot)\right), \cdots, g_{n}\left(x_{n}(\cdot)\right)\right]^{\mathrm{T}} \in \square^{n}$ denotes the neuron activation function with $g(0)=0 ; C=\operatorname{diag}\left\{c_{1}, c_{2}, \cdots, c_{n}\right\}$ is a diagonal matrix representing self-feedback term, $A, B \in \square^{n \times n}$ are the connection weight matrix and the delayed connection weight matrix, respectively, $J=\left[J_{1}, J_{2}, \cdots, J_{n}\right]^{\mathrm{T}} \in \square^{n}$ is a constant input vector. $d_{1}(t)$ and $d_{2}(t)$ are continuous time-varying functions that represent the two delay components in the state which satisfy

$$
0 \leqslant d_{i}(t) \leqslant d_{i}, \quad 0 \leqslant \dot{d}_{i}(t) \leqslant \mu_{i}(i=1,2),
$$

where $d_{i}, \mu_{i}(i=1,2)$ are known constants. Naturally, we denote

$$
\begin{gathered}
d(t)=d_{1}(t)+d_{2}(t), \\
d=d_{1}+d_{2}, \\
\mu=\mu_{1}+\mu_{2} .
\end{gathered}
$$

In addition, it is assumed that each neuron activation function $g_{i}(\cdot)(i=1,2, \cdots, n)$ is bounded and satisfies the following condition

$$
k_{i}^{-} \leqslant \frac{g_{i}\left(s_{1}\right)-g_{i}\left(s_{2}\right)}{s_{1}-s_{2}} \leqslant k_{i}^{+}, \forall s_{1}, s_{2} \in R, s_{1} \neq s_{2}, i=1,2, \cdots, n,
$$

where $k_{i}^{-}, k_{i}^{+}(i=1,2, \cdots, n)$ are known real constants.

It is easy to prove the existence and uniqueness of the equilibrium of system (1) by employing the well-known Brouwers fixed point theorem [1]. Assume that 
$x^{*}=\left[x_{1}^{*}, x_{2}^{*}, \cdots, x_{n}^{*}\right]^{\mathrm{T}}$ is an equilibrium, by choosing the coordinate transformation $z(\cdot)=x(\cdot)-x^{*}$, system (1) can be converted to

$$
\dot{z}(t)=-C z(t)+A f(z(t))+B f\left(z\left(t-d_{1}(t)-d_{2}(t)\right)\right),
$$

where $z(\cdot)=\left[z_{1}(\cdot), z_{2}(\cdot), \cdots, z_{n}(\cdot)\right]^{\mathrm{T}}$ is the state vector of the transformed system, and the transformed neuron activation function is $f(z(\cdot))=\left[f_{1}\left(z_{1}(\cdot)\right), f_{2}\left(z_{2}(\cdot)\right), \cdots, f_{n}\left(z_{n}(\cdot)\right)\right]^{\mathrm{T}}$, where $\left.f_{i}\left(z_{i}(\cdot)\right)=g_{i}\left(z_{i}(\cdot)+x_{i}^{*}\right)-g_{i}\left(x_{i}^{*}\right)(i=1,2, \cdots, n)\right)$. According to (6), one can obtain that

$$
k_{i}^{-} \leqslant \frac{f_{i}\left(z_{i}(t)\right)}{z_{i}(t)} \leqslant k_{i}^{+}, f_{i}(0)=0, \forall z_{i}(t) \neq 0,
$$

which implies that

$$
\begin{gathered}
{\left[f_{i}\left(z_{i}(t)\right)-k_{i}^{-} z_{i}(t)\right]\left[f_{i}\left(z_{i}(t)\right)-k_{i}^{+} z_{i}(t)\right] \leqslant 0,} \\
{\left[f_{i}\left(z_{i}(t-d(t))\right)-k_{i}^{-} z_{i}(t-d(t))\right]\left[f_{i}\left(z_{i}(t-d(t))\right)-k_{i}^{+} z_{i}(t-d(t))\right] \leqslant 0,}
\end{gathered}
$$

where $t>0$ and $i=1,2, \cdots, n$.

Remark 1 It is easy to see that the assumed conditions (6) and (8) of neuron activation functions for systems (1) and (7) include those used conditions in [31-36] as their special cases when $k_{i}^{-}=0$ and $k_{i}^{+}>0$.

To the end this section, we introduce the following lemmas, which will play an important role in the proof of the main results.

Lemma 1 (Jensen inequality [3]) For any matrix $R>0$, scalars $\gamma_{1}$ and $\gamma_{2}$ satisfying $\gamma_{2}>\gamma_{1}$, a vector function $\omega:\left[\gamma_{1}, \gamma_{2}\right] \rightarrow \square{ }^{n}$ such that the integrations concerned are well defined, then the following inequality holds

$$
-\left(\gamma_{2}-\gamma_{1}\right) \int_{\gamma_{1}}^{\gamma_{2}} \omega^{\mathrm{T}}(s) R \omega(s) \mathrm{d} s \leqslant-\int_{\gamma_{1}}^{\gamma_{2}} \omega^{\mathrm{T}}(s) \mathrm{d} s R \int_{\gamma_{1}}^{\gamma_{2}} \omega(s) \mathrm{d} s .
$$

Lemma 2 (Reciprocally convex lemma [4]) For any real numbers $\alpha_{1}>0, \alpha_{2}>0$ with $\alpha_{1}+\alpha_{2}=1$, vectors $\zeta_{1}, \zeta_{2}$, and matrices $R>0, M$ satisfying

$$
\left[\begin{array}{cc}
R & M \\
M^{\mathrm{T}} & R
\end{array}\right] \geqslant 0
$$

then the following inequality holds

$$
-\frac{1}{\alpha_{1}} \zeta_{1}{ }^{\mathrm{T}} R \zeta_{1}-\frac{1}{\alpha_{2}} \zeta_{2}{ }^{\mathrm{T}} R \zeta_{2} \leqslant-\left[\begin{array}{l}
\zeta_{1} \\
\zeta_{2}
\end{array}\right]^{\mathrm{T}}\left[\begin{array}{cc}
R & M \\
M^{\mathrm{T}} & R
\end{array}\right]\left[\begin{array}{l}
\zeta_{1} \\
\zeta_{2}
\end{array}\right] .
$$




\section{Main Results}

In this sequel, a more general type of Lyapunov functional is proposed to derive new delay delay-dependent stability criteria for neural network (7) with additive time-varying delay components satisfying (2). Before introducing the main results, following notations are defined for simplicity.

$$
\begin{aligned}
& K_{1}=\operatorname{diag}\left\{k_{1}^{-}, k_{2}^{-}, \cdots, k_{n}^{-}\right\}, K_{2}=\operatorname{diag}\left\{k_{1}^{+}, k_{2}^{+}, \cdots, k_{n}^{+}\right\}, \\
& \boldsymbol{\Sigma}_{1}=\operatorname{diag}\left\{k_{1}^{-} k_{1}^{+}, k_{2}^{-} k_{2}^{+}, \cdots, k_{n}^{-} k_{n}^{+}\right\}, \quad \boldsymbol{\Sigma}_{2}=\operatorname{diag}\left\{k_{1}^{-}+k_{1}^{+}, k_{2}^{-}+k_{2}^{+}, \cdots, k_{n}^{-}+k_{n}^{+}\right\} \text {, } \\
& \xi(t)=\left[\begin{array}{lllllll}
z^{\mathrm{T}}(t) & f^{\mathrm{T}}(z(t)) & f^{\mathrm{T}}(z(t-d(t))) & z^{\mathrm{T}}\left(t-d_{1}\right) & z^{\mathrm{T}}\left(t-d_{2}\right) & z^{\mathrm{T}}\left(t-d_{1}(t)\right) & z^{\mathrm{T}}\left(t-d_{2}(t)\right)
\end{array}\right. \\
& \left.z^{\mathrm{T}}(t-d(t)) \quad z^{\mathrm{T}}\left(t-d_{1}(t)-d_{2}\right) \quad z^{\mathrm{T}}\left(t-d_{1}-d_{2}(t)\right) \quad z^{\mathrm{T}}(t-d)\right]^{\mathrm{T}},, \\
& e_{0}=-C e_{1}+A e_{2}+B e_{3}, \quad e_{i}=\left[\begin{array}{lll}
0_{n \times(i-1) n} & I_{n} & 0_{n \times(11-i) n}
\end{array}\right](i=1,2, \cdots, 11) \text {. }
\end{aligned}
$$

In the following, we present a new stability criterion.

Theorem 1 For given scalars $d_{i}>0, \mu_{i}>0(i=1,2)$, the system (7) with two additive time-varying delay components satisfying (2) is globally asymptotically stable if there exist matrices $P>0, Q_{i}>0(i=1,2, \cdots, 9), R_{1}>0, R_{2}>0$, positive diagonal matrices $\Lambda_{j}=\operatorname{diag}\left\{\lambda_{j 1}, \lambda_{j 2}, \cdots, \lambda_{j n}\right\} \geqslant 0, \Delta_{j}=\operatorname{diag}\left\{\delta_{j 1}, \delta_{j 2}, \cdots, \delta_{j n}\right\} \geqslant 0(j=1,2) \quad, \quad$ and matrices $M_{k}(k=1,2,3,4)$ with appropriate dimensions such that the following LMIs hold

$$
\begin{aligned}
& \Xi=\Xi_{1}+\Xi_{2}+\Xi_{3}+\Xi_{4}<0, \\
& {\left[\begin{array}{cc}
R_{1} & \left.M_{i}\right\rceil \\
M_{i}{ }^{\mathrm{T}} & R_{1}
\end{array}\right] \geqslant 0(i=1,2),} \\
& \left\lceil\begin{array}{cc}
R_{2} & M_{j} \\
M_{j}{ }^{\mathrm{T}} & R_{2}
\end{array}\right\rfloor \geqslant 0(j=1,2),
\end{aligned}
$$

where

$$
\begin{aligned}
& \Xi_{1}=e_{1}^{\mathrm{T}}\left(P-K_{1} \Lambda_{1}+K_{2} \Lambda_{2}\right) e_{0}+e_{0}^{\mathrm{T}}\left(P-\Lambda_{1} K_{1}+\Lambda_{2} K_{2}\right) e_{1}+e_{2}^{\mathrm{T}}\left(\Lambda_{1}-\Lambda_{2}\right) e_{0}+e_{0}^{\mathrm{T}}\left(\Lambda_{1}-\Lambda_{2}\right) e_{2}, \\
& \Xi_{2}=e_{1}{ }^{\mathrm{T}}\left(Q_{1}+Q_{2}+Q_{3}+Q_{4}+Q_{5}\right) e_{1}+e_{2}{ }^{\mathrm{T}} Q_{6} e_{2}-(1-\mu) e_{3}{ }^{\mathrm{T}} Q_{6} e_{3}+e_{4}{ }^{\mathrm{T}}\left(-Q_{1}+Q_{8}\right) e_{4}+e_{5}{ }^{\mathrm{T}}\left(-Q_{2}+Q_{7}\right) e_{5} \\
& -\left(1-\mu_{1}\right) e_{6}{ }^{\mathrm{T}} Q_{3} e_{6}-\left(1-\mu_{2}\right) e_{7}{ }^{\mathrm{T}} Q_{4} e_{7}-(1-\mu) e_{8}{ }^{\mathrm{T}} Q_{5} e_{8}-\left(1-\mu_{1}\right) e_{9}{ }^{\mathrm{T}} Q_{7} e_{9}-\left(1-\mu_{2}\right) e_{10}{ }^{\mathrm{T}} Q_{8} e_{10}-e_{11}{ }^{\mathrm{T}} Q_{9} e_{11}, \\
& \Xi_{3}=d e_{0}^{\mathrm{T}}\left(R_{1}+R_{2}\right) e_{0}
\end{aligned}
$$

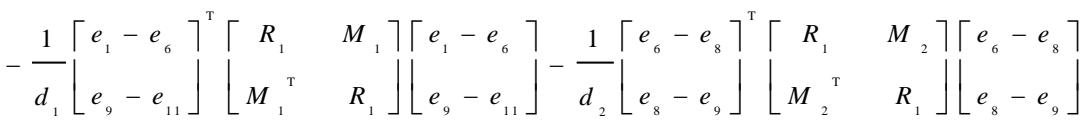

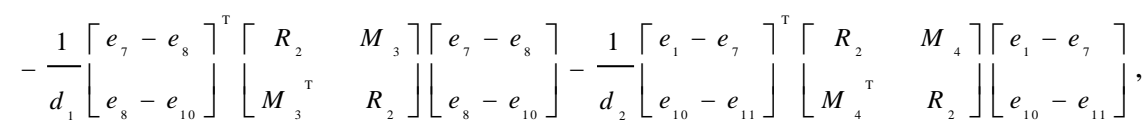

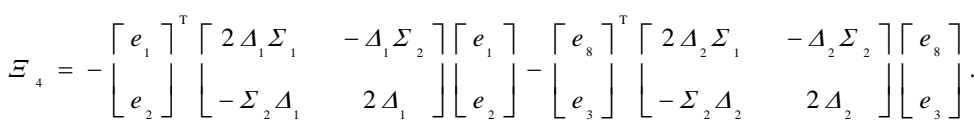

Proof Construct a new class of Lyapunov functional as follow

$$
V(z(t))=\sum^{3} V_{i}(z(t)),
$$

where

$$
V_{1}(z(t))=z^{\mathrm{T}}(t) P z(t)+2 \sum_{i=1}^{n} \int_{0}^{z_{i}(t)}\left[\lambda_{1 i}\left(f_{i}(s)-k_{i}^{-} s\right)+\lambda_{2 i}\left(k_{i}^{+} s-f_{i}(s)\right)\right] \mathrm{d} s,
$$




$$
\begin{aligned}
V_{2}(z(t))= & \int_{t-d_{1}}^{t} z^{\mathrm{T}}(s) Q_{1} z(s) \mathrm{d} s+\int_{t-d_{2}}^{t} z^{\mathrm{T}}(s) Q_{2} z(s) \mathrm{d} s+\int_{t-d_{1}(t)}^{t} z^{\mathrm{T}}(s) Q_{3} z(s) \mathrm{d} s+\int_{t-d_{2}(t)}^{t} z^{\mathrm{T}}(s) Q_{4} z(s) \mathrm{d} s \\
& +\int_{t-d(t)}^{t}\left(z^{\mathrm{T}}(s) Q_{5} z(s)+f^{\mathrm{T}}(z(s)) Q_{6} f(z(s))\right) \mathrm{d} s+\int_{t-d(t)-d_{2}}^{t-d_{2}} z^{\mathrm{T}}(s) Q_{7} z(s) \mathrm{d} s \\
& +\int_{t-d_{1}-d_{2}(t)}^{t-d_{1}} z^{\mathrm{T}}(s) Q_{8} z(s) \mathrm{d} s+\int_{t-d}^{t} z^{\mathrm{T}}(s) Q_{9} z(s) \mathrm{d} s, \\
V_{3}(z(t))= & \int_{-d}^{0} \int_{t+\theta}^{t} \dot{z}^{\mathrm{T}}(s)\left(R_{1}+R_{2}\right) \dot{z}(s) \mathrm{d} s \mathrm{~d} \theta .
\end{aligned}
$$

Taking the time-derivatives of functionals $V_{i}(z(t))(i=1,2,3)$ along the trajectories of system (7), we have

$$
\begin{aligned}
& \dot{V}_{1}(z(t))=2 z^{\mathrm{T}}(t) P \dot{z}(t)+2 \sum\left[\lambda_{1 i}\left(f_{i}\left(z_{i}(t)\right)-k_{i}^{-} z_{i}(t)\right)+\lambda_{2 i}\left(k_{i}^{+} z_{i}(t)-f_{i}\left(z_{i}(t)\right)\right)\right] \dot{z}(t) \\
& =2 z^{\mathrm{T}}(t)\left(P-K_{1} \Lambda_{1}+K_{2} \Lambda_{2}\right) \dot{z}(t)+2 f^{\mathrm{T}}(z(t))\left(\Lambda_{1}-\Lambda_{2}\right) \dot{z}(t) \\
& =\xi^{\mathrm{T}}(t) \Xi_{1} \xi(t) \text {. } \\
& \dot{V}_{2}(z(t))=z^{\mathrm{T}}(t)\left(Q_{1}+Q_{2}+Q_{3}+Q_{4}+Q_{5}+Q_{9}\right) z(t)+f^{\mathrm{T}}(z(t)) Q_{6} f(z(t)) \\
& -(1-\dot{d}(t)) f^{\mathrm{T}}(z(t-d(t))) Q_{6} f(z(t-d(t)))+z^{\mathrm{T}}\left(t-d_{1}\right)\left(-Q_{1}+Q_{8}\right) z\left(t-d_{1}\right) \\
& +z^{\mathrm{T}}\left(t-d_{2}\right)\left(-Q_{2}+Q_{7}\right) z\left(t-d_{2}\right)-\left(1-d_{1}(t)\right) z^{\mathrm{T}}\left(t-d_{1}(t)\right) Q_{3} z\left(t-d_{1}(t)\right) \\
& -\left(1-\dot{d}_{2}(t)\right) z^{\mathrm{T}}\left(t-d_{2}(t)\right) Q_{4} z\left(t-d_{2}(t)\right)-(1-\dot{d}(t)) z^{\mathrm{T}}(t-d(t)) Q_{5} z(t-d(t)) \\
& -\left(1-\dot{d}_{1}(t)\right) z^{\mathrm{T}}\left(t-d_{1}(t)-d_{2}\right) Q_{7} z\left(t-d_{1}(t)-d_{2}\right) \\
& -\left(1-\dot{d}_{2}(t)\right) z^{\mathrm{T}}\left(t-d_{1}-d_{2}(t)\right) Q_{8} z\left(t-d_{1}-d_{2}(t)\right)-z^{\mathrm{T}}(t-d) Q_{9} z(t-d) \\
& \leqslant z^{\mathrm{T}}(t)\left(Q_{1}+Q_{2}+Q_{3}+Q_{4}+Q_{5}+Q_{9}\right) z(t)+f^{\mathrm{T}}(z(t)) Q_{6} f(z(t)) \\
& -(1-\mu) f^{\mathrm{T}}(z(t-d(t))) Q_{6} f(z(t-d(t)))+z^{\mathrm{T}}\left(t-d_{1}\right)\left(-Q_{1}+Q_{8}\right) z\left(t-d_{1}\right) \\
& +z^{\mathrm{T}}\left(t-d_{2}\right)\left(-Q_{2}+Q_{7}\right) z\left(t-d_{2}\right)-\left(1-\mu_{1}\right) z^{\mathrm{T}}\left(t-d_{1}(t)\right) Q_{3} z\left(t-d_{1}(t)\right) \\
& -\left(1-\mu_{2}\right) z^{\mathrm{T}}\left(t-d_{2}(t)\right) Q_{4} z\left(t-d_{2}(t)\right)-(1-\mu) z^{\mathrm{T}}(t-d(t)) Q_{5} z(t-d(t)) \\
& -\left(1-\mu_{1}\right) z^{\mathrm{T}}\left(t-d_{1}(t)-d_{2}\right) Q_{7} z\left(t-d_{1}(t)-d_{2}\right) \\
& -\left(1-\mu_{2}\right) z^{\mathrm{T}}\left(t-d_{1}-d_{2}(t)\right) Q_{8} z\left(t-d_{1}-d_{2}(t)\right)-z^{\mathrm{T}}(t-d) Q_{9} z(t-d) \\
& =\xi^{\mathrm{T}}(t) \Xi_{2} \xi(t) \\
& \dot{V}_{3}(z(t))=d \dot{z}^{\mathrm{T}}(t)\left(R_{1}+R_{2}\right) \dot{z}(t)-\int_{t-d}^{t} \dot{z}^{\mathrm{T}}(s) R_{1} \dot{z}(s) \mathrm{d} s-\int_{t-d}^{t} \dot{z}^{\mathrm{T}}(s) R_{2} \dot{z}(s) \mathrm{d} s \\
& =d \dot{z}^{\mathrm{T}}(t)\left(R_{1}+R_{2}\right) \dot{z}(t)-\int_{t-d_{1}(t)}^{t} \dot{z}^{\mathrm{T}}(s) R_{1} \dot{z}(s) \mathrm{d} s-\int_{t-d(t)}^{t-d_{1}(t)} \dot{z}^{\mathrm{T}}(s) R_{1} \dot{z}(s) \mathrm{d} s \\
& -\int_{t-d_{1}(t)-d_{2}}^{t-d(t)} \dot{z}^{\mathrm{T}}(s) R_{1} \dot{z}(s) \mathrm{d} s-\int_{t-d}^{t-d_{1}(t)-d_{2}} \dot{z}^{\mathrm{T}}(s) R_{1} \dot{z}(s) \mathrm{d} s-\int_{t-d_{2}(t)}^{t} \dot{z}^{\mathrm{T}}(s) R_{2} \dot{z}(s) \mathrm{d} s \\
& -\int_{t-d(t)}^{t-d_{2}(t)} \dot{z}^{\mathrm{T}}(s) R_{2} \dot{z}(s) \mathrm{d} s-\int_{t-d_{1}-d d_{2}(t)}^{t-d(t)} \dot{z}^{\mathrm{T}}(s) R_{2} \dot{z}(s) \mathrm{d} s-\int_{t-d}^{t-d_{1}-d_{2}(t)} \dot{z}^{\mathrm{T}}(s) R_{2} \dot{z}(s) \mathrm{d} s .
\end{aligned}
$$

\section{By Lemma 1, one can obtain}

$$
\begin{aligned}
\dot{V}_{3}(z(t)) & \leqslant d \dot{z}^{\mathrm{T}}(t)\left(R_{1}+R_{2}\right) \dot{z}(t) \\
& -\frac{1}{d_{1}(t)}\left[\int_{t-d_{1}(t)}^{t} \dot{z}(s) \mathrm{d} s\right]^{\mathrm{T}} R_{1}\left[\int_{t-d_{1}(t)}^{t} \dot{z}(s) \mathrm{d} s\right]-\frac{1}{d_{1}-d_{1}(t)}\left[\int_{t-d}^{t-d_{1}(t)-d_{2}} \dot{z}(s) \mathrm{d} s\right]^{\mathrm{T}} R_{1}\left[\int_{t-d}^{t-d_{1}(t)-d_{2}} \dot{z}(s) \mathrm{d} s\right] \\
& -\frac{1}{d_{2}(t)}\left[\int_{t-d(t)}^{t-d_{1}(t)} \dot{z}(s) \mathrm{d} s\right]^{\mathrm{T}} R_{1}\left[\int_{t-d(t)}^{t-d_{1}(t)} \dot{z}(s) \mathrm{d} s\right]-\frac{1}{d_{2}-d_{2}(t)}\left[\int_{t-d_{1}(t)-d_{2}}^{t-d(t)} \dot{z}(s) \mathrm{d} s\right]^{\mathrm{T}} R_{1}\left[\int_{t-d_{1}(t)-d_{2}}^{t-d(t)} \dot{z}(s) \mathrm{d} s\right]
\end{aligned}
$$




$$
\begin{aligned}
& -\frac{1}{d_{1}(t)}\left[\int_{t-d(t)}^{t-d_{2}(t)} \dot{z}(s) \mathrm{d} s\right]^{\mathrm{T}} R_{2}\left[\int_{t-d(t)}^{t-d_{2}(t)} \dot{z}(s) \mathrm{d} s\right]-\frac{1}{d_{1}-d_{1}(t)}\left[\int_{t-d_{1}-d_{2}(t)}^{t-d(t)} \dot{z}(s) \mathrm{d} s\right]^{\mathrm{T}} R_{2}\left[\int_{t-d_{1}-d_{2}(t)}^{t-d(t)} \dot{z}(s) \mathrm{d} s\right] \\
& -\frac{1}{d_{2}(t)}\left[\int_{t-d_{2}(t)}^{t} \dot{z}(s) \mathrm{d} s\right]^{\mathrm{T}} R_{2}\left[\int_{t-d_{2}(t)}^{t} \dot{z}(s) \mathrm{d} s\right]-\frac{1}{d_{2}-d_{2}(t)}\left[\int_{t-d}^{t-d_{1}-d_{2}(t)} \dot{z}(s) \mathrm{d} s\right]^{\mathrm{T}} R_{2}\left[\int_{t-d}^{t-d_{1}-d_{2}(t)} \dot{z}(s) \mathrm{d} s\right] \\
= & d \xi^{\mathrm{T}}(t) e_{0}^{\mathrm{T}}\left(R_{1}+R_{2}\right) e_{0} \xi(t) \\
& -\frac{1}{d_{1}} \frac{1}{\alpha_{1}} \xi^{\mathrm{T}}(t)\left(e_{1}-e_{6}\right)^{\mathrm{T}} R_{1}\left(e_{1}-e_{6}\right) \xi(t)-\frac{1}{d_{1}} \frac{1}{\alpha_{2}} \xi^{\mathrm{T}}(t)\left(e_{9}-e_{11}\right)^{\mathrm{T}} R_{1}\left(e_{9}-e_{11}\right) \xi(t) \\
& -\frac{1}{d_{2}} \frac{1}{\alpha_{3}} \xi^{\mathrm{T}}(t)\left(e_{6}-e_{8}\right)^{\mathrm{T}} R_{1}\left(e_{6}-e_{8}\right) \xi(t)-\frac{1}{d_{2}} \frac{1}{\alpha_{4}} \xi^{\mathrm{T}}(t)\left(e_{8}-e_{9}\right)^{\mathrm{T}} R_{1}\left(e_{8}-e_{9}\right) \xi(t) \\
& -\frac{1}{d_{1}} \frac{1}{\alpha_{1}} \xi^{\mathrm{T}}(t)\left(e_{7}-e_{8}\right)^{\mathrm{T}} R_{2}\left(e_{7}-e_{8}\right) \xi(t)-\frac{1}{d_{1}} \frac{1}{\alpha_{2}} \xi^{\mathrm{T}}(t)\left(e_{8}-e_{10}\right)^{\mathrm{T}} R_{2}\left(e_{8}-e_{10}\right) \xi(t) \\
& -\frac{1}{d_{2}} \frac{1}{\alpha_{3}} \xi^{\mathrm{T}}(t)\left(e_{1}-e_{7}\right)^{\mathrm{T}} R_{2}\left(e_{1}-e_{7}\right) \xi(t)-\frac{1}{d_{2}} \frac{1}{\alpha_{4}} \xi^{\mathrm{T}}(t)\left(e_{10}-e_{11}\right)^{\mathrm{T}} R_{2}\left(e_{10}-e_{11}\right) \xi(t),
\end{aligned}
$$

where

$$
\alpha_{1}=\frac{d_{1}(t)}{d_{1}}, \alpha_{2}=\frac{d_{1}-d_{1}(t)}{d_{1}}, \alpha_{3}=\frac{d_{2}(t)}{d_{2}}, \alpha_{4}=\frac{d_{2}-d_{2}(t)}{d_{2}} .
$$

From Lemma 2 and LMIs (12)、(13), we have

$$
\dot{V}_{3}(z(t)) \leqslant \xi^{\mathrm{T}}(t) \Xi_{3} \xi(t) .
$$

On the other hand, from (9) and (10), it yields that

$$
\begin{aligned}
& 0 \leqslant-2 \sum_{i=1}^{n} \delta_{1 i}\left[f_{i}\left(z_{i}(t)\right)-k_{i}^{-} z_{i}(t)\right]\left[f_{i}\left(z_{i}(t)\right)-k_{i}^{+} z_{i}(t)\right]
\end{aligned}
$$

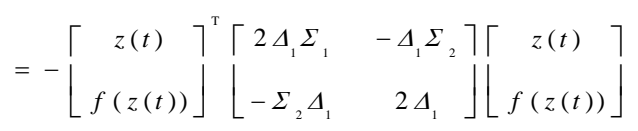

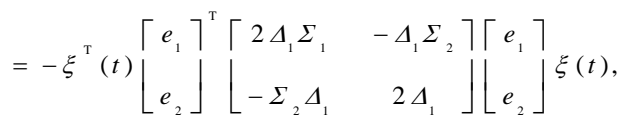

and

$$
\begin{aligned}
& 0 \leqslant-2 \sum^{n} \delta_{2 i}\left[f_{i}\left(z_{i}(t-d(t))\right)-k_{i}^{-} z_{i}(t-d(t))\right]\left[f_{i}\left(z_{i}(t-d(t))\right)-k_{i}^{+} z_{i}(t-d(t))\right]
\end{aligned}
$$

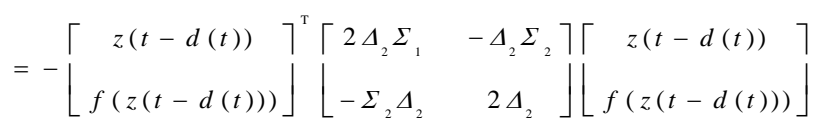

$$
\begin{aligned}
& =-\xi^{\mathrm{T}}(t)\left[\begin{array}{l}
e_{8} \\
e_{3}
\end{array}\right]^{\mathrm{T}}\left[\begin{array}{cc}
2 \Delta_{2} \Sigma_{1} & \left.-\Delta_{2} \Sigma_{2}\right\rceil\left\lceil e_{8}\right. \\
-\Sigma_{2} \Delta_{2} & 2 \Delta_{2}
\end{array}\right]\left[\begin{array}{l}
e_{3}
\end{array}\right] \xi(t) .
\end{aligned}
$$

Hence, according to (15)-(19), we can obtain

$$
\dot{V}(z(t)) \leqslant \xi^{\mathrm{T}}(t) \Xi \xi(t) .
$$

Thus, system (7) is globally asymptotically stable. This completes the proof.

Remark 2 Theorem 1 provides a novel delay-dependent stability criteria for neural networks with two additive time-varying delay components. It is noted that unlike the Lyapunov functionals chosen in [31-36], the constructed Lyapunov functional (14) takes the states $z\left(t-d_{1}(t)-d_{2}\right)$ and $z\left(t-d_{1}-d_{2}(t)\right)$ into account, which make full use of the information of the neuron activation functions and the involved time-varying delay. So, 
the Lyapunov functional in our paper is more general than that in [31-36], and the stability criterion in our paper may be less conservative.

Remark 3 It is worth mentioning that in [31-36], the free-weighting matrices technique is utilized to discuss the stability problem for neural networks with two additive time-varying delay components. However, some matrices do not reduce the conservatism of the resulting criterion; on the contrary, they make the stability analysis more complicated and lead to a significant increase in the computational demand. While in our proof, we enlarge the cross terms appeared in the derivative of Lyapunov functional by using reciprocally convex method [5]. Thus, the criterion derived in Theorem 1 has less decision variables than the ones in [31-36].

When the informations of the derivative of time-varying delays are unknown, a rateindependent corollary for the delays $d_{1}(t)$ and $d_{2}(t)$ satisfying $0 \leqslant d_{i}(t) \leqslant d_{i}(i=1,2)$ is derived by setting $Q_{j}=0(j=3,4, \cdots, 8)$ in the proof of Theorem 1 .

Corollary 1 For given scalars $d_{i}>0(i=1,2)$, the system (7) with two additive timevarying delay components satisfying $0 \leqslant d_{i}(t) \leqslant d_{i}(i=1,2)$ is globally asymptotically stable if there exist matrices $P>0, Q_{1}>0, Q_{2}>0, Q_{9}>0, R_{1}>0, R_{2}>0$, positive diagonal matrices $\Lambda_{j}=\operatorname{diag}\left\{\lambda_{j 1}, \lambda_{j 2}, \cdots, \lambda_{j n}\right\} \geqslant 0 \quad, \quad \Delta_{j}=\operatorname{diag}\left\{\delta_{j 1}, \delta_{j 2}, \cdots, \delta_{j n}\right\} \geqslant 0(j=1,2) \quad, \quad$ and matrices $M_{k}(k=1,2,3,4)$ with appropriate dimensions such that the LMIs (12),(13) and following LMI hold

where

$$
\Xi_{1}+\tilde{\Xi}_{2}+\Xi_{3}+\Xi_{4}<0
$$

$$
\tilde{\Xi}_{2}=e_{1}^{\mathrm{T}}\left(Q_{1}+Q_{2}+Q_{9}\right) e_{1}-e_{4}^{\mathrm{T}} Q_{1} e_{4}-e_{5}^{\mathrm{T}} Q_{2} e_{5}-e_{11}^{\mathrm{T}} Q_{9} e_{11},
$$

and

$\Xi_{1}, \Xi_{3}, \Xi_{4}$ are defined as in Theorem 1 .

Remark 4 It is worth pointing out that the obtained results in this paper are all formulated in terms of LMIs, they can be easily solved using any LMI toolbox like one of Matlab.

\section{Numerical Examples}

In this section, two numerical examples are given to illustrate the effectiveness and the less conservatism of obtained results in this paper.

Example 1 Consider the neural network (7) with following parameters

$$
\begin{gathered}
C=\left[\begin{array}{cc}
2.0 & 0 \\
0 & 1.8
\end{array}\right], A=\left[\begin{array}{cc}
1.2 & 1.0 \\
-1.0 & -1.2
\end{array}\right], B=\left[\begin{array}{cc}
1.0 & 1.0 \\
-1.0 & 0.8
\end{array}\right], \\
K_{1}=\operatorname{diag}\{-0.2,-0.4\}, K_{2}=\operatorname{diag}\{0.4,0.8\} .
\end{gathered}
$$

Case I : For given $d_{1}=1.0, \mu_{1}=0.3, \mu_{2}=0.6$, by resorting to the Matlab LMI toolbox to solve the LMIs in Theorem 1, it is concluded that system (7) is globally asymptotically stable while $d_{2}$ is up to 1.6620 , and the corresponding feasible solutions of LMIs (11)(13) are given as follows

$$
P=\left[\begin{array}{ll}
340.3995 & 29.5773 \\
29.5773 & 437.4800
\end{array}\right], Q_{1}=\left[\begin{array}{ll}
290.8926 & 92.4557 \\
92.4557 & 30.0660
\end{array}\right], Q_{2}=\left[\begin{array}{cc}
7.6643 & -29.1050 \\
-29.1050 & 126.2785
\end{array}\right],
$$




$$
\begin{aligned}
& Q_{3}=\left[\begin{array}{cc}
6.9822 & -27.6835 \\
-27.6835 & 127.94448
\end{array}\right], Q_{4}=\left[\begin{array}{ll}
290.2311 & 90.5342 \\
90.5342 & 29.0593
\end{array}\right], Q_{5}=\left[\begin{array}{ll}
0.0233 & 0.0128 \\
0.0128 & 0.0110
\end{array}\right], \\
& Q_{6}=10^{3} \times\left[\begin{array}{cc}
0.5363 & -1.3635 \\
-1.3635 & 3.4672
\end{array}\right], Q_{7}=\left[\begin{array}{cc}
7.6619 & -29.1063 \\
-29.1063 & 126.2754
\end{array}\right], Q_{8}=\left[\begin{array}{cc}
290.8902 & 92.4543\rceil \\
92.4543 & 30.0629
\end{array}\right], \\
& Q_{9}=\left[\begin{array}{cc}
74.0150 & 6.8621 \\
6.8621 & 94.9614
\end{array}\right], R_{1}=\left[\begin{array}{cc}
9.4331 & -38.8144 \\
-38.8144 & 173.5194
\end{array}\right], R_{2}=\left[\begin{array}{ll}
92.1490 & 28.8032 \\
28.8032 & 11.1457
\end{array}\right], \\
& \cdot \Lambda_{1}=\left[\begin{array}{cc}
445.1171 & 0 \\
0 & 917.0683
\end{array}\right], \Lambda_{2}=\left[\begin{array}{cc}
260.9814 & 0 \\
0 & 242.2099
\end{array}\right], \Delta_{1}=10^{3} \times\left[\begin{array}{cc}
2.3534 & 0 \\
0 & 1.9197
\end{array}\right] \text {, } \\
& \Delta_{2}=\left[\begin{array}{cc}
739.4622 & 0 \\
0 & 212.7990
\end{array}\right], M_{1}=\left[\begin{array}{cc}
-8.8791 & 39.7223 \\
32.9453 & -156.8078
\end{array}\right], M_{2}=\left[\begin{array}{cc}
-9.4320 & 38.9257 \\
38.6997 & -173.5083
\end{array}\right], \\
& M_{3}=\left[\begin{array}{ll}
-92.1470 & -28.7258 \\
-28.8797 & -11.1387
\end{array}\right], M_{4}=\left[\begin{array}{ll}
-91.4163 & -26.8136 \\
-30.3343 & -11.0515
\end{array}\right] \text {. }
\end{aligned}
$$

Case II : For given $d_{1}=1.0$ and unknown $\mu_{1}, \mu_{2}$, by resorting to the Matlab LMI toolbox to solve the LMIs in Corollary 1 , it is concluded that system (7) is globally asymptotically stable while $d_{2}$ is up to 0.9669 , and the corresponding feasible solutions of LMIs (12),(13),(20) are given as follows

$$
\begin{aligned}
& P=\left[\begin{array}{ll}
869.0976 & -29.8226 \\
-29.8226 & 999.4159
\end{array}\right], Q_{1}=Q_{2}=\left[\begin{array}{ll}
0.0655 & 0.0120 \\
0.0120 & 0.0484
\end{array}\right], Q_{9}=10^{3} \times\left[\begin{array}{ll}
0.9417 & 0.1586 \\
0.1586 & 1.4051
\end{array}\right], \\
& R_{1}=\left[\begin{array}{ll}
206.3890 & -43.2173 \\
-43.2173 & 400.7494
\end{array}\right], R_{2}=\left[\begin{array}{ll}
207.5793 & -43.4705 \\
-43.4705 & 403.0807
\end{array}\right], \Lambda_{1}=10^{3} \times\left[\begin{array}{cc}
1.2371 & 0 \\
0 & 2.9284
\end{array}\right], \\
& \text { - } \Lambda_{2}=\left[\begin{array}{ll}
870.7120 & -43.2173 \\
-43.2173 & 648.6941
\end{array}\right], \Delta_{1}=10^{3} \times\left[\begin{array}{cc}
8.0968 & 0 \\
0 & 1.6775
\end{array}\right], \Delta_{2}=10^{3} \times\left[\begin{array}{cc}
2.3738 & 0 \\
0 & 1.1019
\end{array}\right] \text {, } \\
& \text { - } M_{1}=\left[\begin{array}{cc}
-206.1429 & 56.4754 \\
29.8478 & -400.2845
\end{array}\right], M_{2}=\left[\begin{array}{cc}
-206.1415 & 56.4748 \\
29.8474 & -400.2829
\end{array}\right] \text {, } \\
& M_{3}=\left[\begin{array}{cc}
-207.3318 & 56.8057 \\
30.0234 & -402.6131
\end{array}\right], M_{4}=\left[\begin{array}{cc}
-207.3305 & 56.8052 \\
30.0229 & -402.6116
\end{array}\right] \text {. }
\end{aligned}
$$

Example 2 Consider the neural network (7) with following parameters

$$
\begin{gathered}
C=\left[\begin{array}{cc}
2.0 & 0 \\
0 & 2.0
\end{array}\right], A=\left[\begin{array}{cc}
1.0 & 1.0 \\
-1.0 & -1.0
\end{array}\right], B=\left[\begin{array}{cc}
0.88 & 1.0 \\
1.0 & 1.0
\end{array}\right], \\
K_{1}=\operatorname{diag}\{0,0\}, K_{2}=\operatorname{diag}\{0.4,0.8\} .
\end{gathered}
$$

Assuming $\mu_{1}=0.7, \mu_{2}=0.1$. Our purpose is to calculate the upper delay bound $d_{2}$ of delay $d_{2}(t)$, or $d_{1}$ of delay $d_{1}(t)$, when the other is known, below which the system (7) is globally asymptotically stable. The maximum value of upper delay bound $d_{2}$ obtained by [32,34,35] and Theorem 1 in this paper for different $d_{1}$ are listed in Table I. The maximum value of upper delay bound $d_{1}$ obtained by $[31,33,36]$ and Theorem 1 in this paper for different $d_{2}$ are listed in Table II. From Table I and Table II we can see that by using Theorem 1, our obtained results are less conservative than those of [31-36].

Moreover, Theorem 1 in this paper contain fewer decision variables than those conditions in [31-36], which can be seen from Table III. Hence, it is mathematically less complex and computationally more efficient. 
Table 1. Allowable Upper Bound $d_{2}$ for Various $d_{1}$

\begin{tabular}{|c|c|c|c|}
\hline Methods & $d_{1}=0.8$ & $d_{1}=1.0$ & $d_{1}=1.2$ \\
\hline$[32]$ & 1.5666 & 1.3668 & 1.1664 \\
\hline$[34]$ & 2.0164 & 1.8203 & 1.6197 \\
\hline$[35]$ & 2.0961 & 1.8961 & 1.6961 \\
\hline Theorem 1 & 5.0811 & 3.7229 & 2.7561 \\
\hline
\end{tabular}

Table 2. Allowable Upper Bound $d_{1}$ for Various $d_{2}$

\begin{tabular}{|c|c|c|c|}
\hline Methods & $d_{2}=0.1$ & $d_{2}=0.2$ & $d_{2}=0.3$ \\
\hline$[31]$ & 2.1564 & 1.6464 & 1.4365 \\
\hline$[36]$ & 2.2571 & 2.1719 & 2.1074 \\
\hline$[33]$ & 2.7248 & 2.3325 & 2.2187 \\
\hline Theorem 1 & 5.3783 & 3.2321 & 2.5117 \\
\hline
\end{tabular}

Table 3. Number of Decision Variables

\begin{tabular}{|c|c|}
\hline Methods & Number of decision variables \\
\hline$[31]$ & $32.5 n^{2}+8.5 n$ \\
\hline$[32]$ & $36.5 n^{2}+8.5 n$ \\
\hline$[33]$ & $15.5 n^{2}+11.5 n$ \\
\hline$[34]$ & $80.5 n^{2}+8.5 n$ \\
\hline$[35]$ & $21.5 n^{2}+8.5 n$ \\
\hline$[36]$ & $11 n^{2}+10 n$ \\
\hline Theorem 1 & $10 n^{2}+10 n$ \\
\hline
\end{tabular}

\section{Conclusion}

This paper deals with the problem of stability for neural networks with two additive time-varying delay components. By constructing a more general type of Lyapunov functional, and using reciprocally convex method, delay-dependent stability criteria are proposed in terms of LMIs for considered systems. Stability criteria are obtained by using more information of the time-varying delays and considering the relationship between the time-varying delays and their upper delay bounds, these results have been shown to be less conservative than some recently report results. Moreover, the proposed method decreases the computational complexity comparable to some existing methods. Two numerical examples are given to illustrate the applicability of the results. The method used in this paper can be further extended to cope with the robust stability problem for such systems and stability problem for delayed neural networks with interval time-varying delay.

\section{References}

[1] A. Cochocki and R. Unbehauen, "Neural networks for optimization and signal processing", John Wiley and Sons, (1993).

[2] M. Gupta, L. Jin and N. Homma, "Static and dynamic neural networks: from fundamentals to advanced theory", John Wiley and Sons, (2004).

[3] Y. Z. Liu and M. G. Li, "New stability analysis for systems with interval time-varying delay via a delayfractioning approach", International Journal of Control and Automation, vol.7, no.4, (2014), pp.9-18.

[4] P. G. Park, J. W. Ko and C. Jeong, "Reciprocally convex approach to stability of systems with timevarying delays", Automatica, vol.47, no.1, (2011), pp.235-238. 
[5] J. M. Jiao and R. Zhang, "A delay decomposition approach to the stability analysis of singular systems with interval time-Varying delay", Mathematical Problems in Engineering, (2014), pp.1-12.

[6] S. Arik, "Global asymptotic stability of a larger class of neural networks with constant time delay", Physics Letters A, vol.311, no.6, (2003), pp.504-511.

[7] Q. Zhang, X. P. Wei and J. Xu, "Stability analysis for cellular neural networks with variable delays", Chaos, Solitons \& Fractals, vol.28, no.2, (2006), pp.331-336.

[8] V. Singh, "LMI approach to the global robust stability of a larger class of neural networks with delay", Chaos, Solitons \& Fractals, vol.32, no.5, (2007), pp.1927-1934.

[9] Y. He, M. Wu and J. H. She, "An improved global asymptotic stability criterion for delayed cellular neural networks", IEEE Transactions on Neural Networks, vol.17, no.1, (2006), pp.250-252.

[10] Y. He, G. P. Liu and D. Rees, "New delay-dependent stability criteria for neural networks with timevarying delay", IEEE Transactions on Neural Networks, vol.18, no.1, (2007), pp.310-314.

[11] C. Y. Lu, H. H. Tsai, T. J. Su, J. S. H. Tsai and C. W. Liao, "A delay-dependent approach to passivity analysis for uncertain neural networks with time-varying delay", Neural Processing Letters, vol.27, no.3, (2008), pp.237-246.

[12] J. K. Tian and S. M. Zhong, "New delay-dependent exponential stability criteria for neural networks with discrete and distributed time-varying delays", Neurocomputing, vol.74, no.17, (2011), pp.3365-3375.

[13] X. H. Liu and H. S. Xi, "Stability analysis for uncertain neutral markovian jump systems with interval time-varying delay", International Journal of Control and Automation, vol.6, no.4, (2013), pp.69-81.

[14] T. Li and X. L. Ye, "Improved stability criteria of neural networks with time-varying delays: an augmented LKF approach”, Neurocomputing, vol.73, no.4, (2010), pp.1038-1047.

[15] C. C. Hua, X. Yang, J. Yan and X. Guan, "New stability criteria for neural networks with time-varying delays", Applied Mathematics and Computation, vol.218, no.9, (2012), pp.5035-5042.

[16] O. M. Kwon, S. M. Lee, J. H. Park and E. J. Cha, "New approaches on stability criteria for neural networks with interval time-varying delays", Applied Mathematics and Computation, vol.218, no.19, (2012), pp.9953-9964.

[17] W. H. Chen, X. M. Lu, Z. H. Guan and W. X. Zheng, "Delay-dependent exponential stability of neural networks with variable delay: an LMI approach", IEEE Transactions on Circuits and Systems II, vol.53, no.9, (2006), pp.837-842.

[18] X. M. Zhang and Q. L. Han, "New Lyapunov-Krasovskii functionals for global asymptotic stability of delayed neural networks", IEEE Transactions on Neural Networks, vol.20, no.3, (2009), pp.533-539.

[19] Y. J. Zhang, D. Yue and E. G. Tian, "New stability criteria of neural networks with interval time-varying delay: a piecewise delay method", Applied Mathematics and Computation, vol.208, no.1, (2009), pp.249-259.

[20] H. B. Zeng, Y. He, M. Wu and C. F. Zhang, "Complete delay-decomposing approach to asymptotic stability for neural networks with time-varying delays", IEEE Transactions on Neural Networks, vol.22, no.5, (2011), pp.806-812.

[21] S. Lakshmanan, J. H. Park, H. Y. Jung and O. M. Kown, "A delay partitioning approach to delaydependent stability analysis for neutral type neural networks with discrete and distributed delays", Neurocomputing, vol.111, (2013), pp.81-89.

[22] C. D. Zheng, C. K. Gong and Z. S. Wang, "New passivity conditions with fewer slack variables for uncertain neural networks with mixed delays", Neurocomputing, vol.118, (2013), pp.237-244.

[23] T. Li, W. X. Zheng and C. Lin, "Delay-slope-dependent stability results of recurrent neural networks", IEEE Transactions on Neural Networks, vol.22, no.12, (2011), pp.2138-2143.

[24] Z. G. Wu, J. H. Park, H. Y. Su and J. Chu, "New results on exponential passivity of neural networks with time-varying delays", Nonlinear Analysis: Real World Applications, vol.13, no.4, (2012), pp.1593-1599.

[25] T. Li, X. Yang, P. Yang and S. M. Fei, "New delay-variation-dependent stability for neural networks with time-varying delay", Neurocomputing, vol.101, (2013), pp.361-369.

[26] J. Lam, H. J. Gao and C. H. Wang, "Stability analysis for continuous systems with two additive timevarying delay components", Systems \& Control Letters, vol.56, no.1, (2007), pp.16-24.

[27] H. J. Gao, T. W. Chen and J. Lam, "A new delay system approach to network-based control", Automatica, vol.44, no.1, (2008), pp.39-52.

[28] H. Y. Shao and Q. L. Han, "On stabilization for systems with two additive time-varying input delays arising from networked control systems", Journal of the Franklin Institute, vol.349, no.6, (2012), pp.2033-2046.

[29] J. M. Jiao, "A stability criterion for singular systems with two additive time-varying delay components", International Journal of Automation and Computing, vol.10, no.1, (2013), pp.39-45.

[30] K. Ramakrishnan and G. Ray, "Stability criteria with less LMI variables for linear system with additive time-delays", Journal of Applied Mathematics and Computing, vol.36, no.1-2, (2011), pp.263-274.

[31] Y. Zhao, H. J. Gao and S. S. Mou, "Asymptotic stability analysis of neural networks with successive time delay components", Neurocomputing, vol.71, no.13, (2008), pp.2848-2856.

[32] H. Y. Shao and Q. L. Han, "New delay-dependent stability criteria for neural networks with two additive time-varying delay components", IEEE Transactions on Neural Networks, vol.22, no.5, (2011), pp.812818. 
[33] N. Xiao and Y. M. Jia, "New approaches on stability criteria for neural networks with two additive timevarying delay components", Neurocomputing, vol.118, (2013), pp.150-156.

[34] J. K. Tian and S. M. Zhong, "Improved delay-dependent stability criteria for neural networks with two additive time-varying delay components", Neurocomputing, vol.77, no.1, (2012), pp.114-119.

[35] H. B. Chen, "Improved stability criteria for neural networks with two additive time-varying delay components", Circuits, Systems, and Signal Processing, vol.32, no.4, (2013), pp.1977-1990.

[36] X. L. Zhu, D. Yue and Y. Y. Wang, "Delay-dependent stability analysis for neural networks with additive time-varying delay components", IET Control Theory \& Applications, vol.7, no.3, (2013), pp.354-362.

\section{Author}

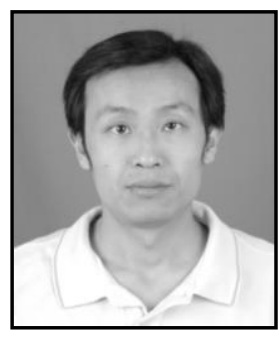

Jianmin Jiao, he received the M.S. in College of Mathematics and Information Science from Shaanxi Normal University, Xi'an, China, in 2009. He is currently an associate professor of College of Mathematics and Information Science, Baoji University of Arts and Sciences, China. His research interests include time-delay systems, neural networks, singular systems and robust control. 
International Journal of Control and Automation Vol. 8, No. 9 (2015) 